\title{
COMPARISON OF "KAMPONG" AND COMMERCIAL CHICKEN EGG-BASED EXTENDERS ON CRYOPRESERVED GOAT SPERM MOVEMENT CHARACTERISTICS
}

\author{
Janice, C.W.K. ${ }^{1}$, Kanwal, K.D.S. ${ }^{1 *}$, Wan Khadijah, W.E. ${ }^{2}$ and Abdullah, R.B. ${ }^{2}$ \\ ${ }^{1}$ School of Sustainable Agriculture, University Malaysia Sabah, Mile 10, Jalan Sg. Batang, 90000 \\ Sandakan, Sabah, Malaysia \\ ${ }^{2}$ Animal Biotechnology-Embryo Laboratory (ABEL), Institute of Biological Sciences, Faculty of Science, \\ University of Malaya, 50603 Kuala Lumpur, Malaysia \\ *Email: keeron_my@yahoo.com (corresponding author)
}

\begin{abstract}
Replacement of egg yolk obtained from commercial chicken with that of kampong chicken egg in the commonly used semen extender, TRIS, was investigated on cryopreserved goat spermatozoa movement characteristics. Fresh and frozen-thawed sperm movement characteristics such as progressive motility, progressive velocity and linearity were analyzed using computer assisted semen analyzer. Sperm samples in the experimental group showed apparently better sperm movement characteristics compared to those cryopreserved in commercial chicken egg yolk, however these differences were non-significant. Based on this preliminary study it could be concluded that kampong egg yolk offers an alternative choice in freezing protocol of goat spermatozoa for artificial insemination.
\end{abstract}

(Keywords: Kampong egg yolk, Semen extender, Computer-assisted sperm analysis, Goat sperm movement characteristics)

\section{INTRODUCTION}

Techniques of mammalian sperm cryopreservation result in loss of sperm viability during freezing-thawing processes even though with significant improvements over the last several decades $[1,2,3]$. The main sources of sperm cryoinjury are cold shock, osmotic stress, ice crystal formation and/or oxidative damage, which finally cause loss of sperm viability and fertility $[4,5,6]$. Sperm cryopreservation in goats causes reduction in sperm motility which could adversely affect the fertility in artificial insemination (AI) programme. Chicken egg yolk and its components can help in providing protection against cold shock [7] and these protective effects are mainly attributed to the low-density lipoprotein (LDL) in the egg yolk [8, 9]. There are reports indicating high survival and fertility rates of bull sperm in extender with $20 \%$ egg yolk [10]. Inclusion of egg yolks from various avian species in the freezing protocols of sperm has also been investigated [11, 12]. Kampong chicken meat and its eggs are quite popular among Malaysian people due to their unique taste, aroma and nutritional qualities and are preferred over those obtained from commercially produced chicken. However, no data is available on the chemical and nutritional composition of kampong chicken egg and its contents. This study was conducted to evaluate the effect of egg yolk of kampong chicken used as cryoprotectant on the cryopreserved and thawed goat sperm movement characteristics.

\section{MATERIAL AND METHODS}

\section{Semen collection and initial evaluation}

Six sexually mature Katjang bucks aged between two and three years maintained at the goat farm, University of Malaya, were used in this study. The healthy bucks were selected based on libido, scrotal circumferences, body weight, and body conformations. The bucks were kept in individual pens and all were fed with the same diet consisting of concentrate pellet and Napier grass. The semen was collected twice a week from each buck using artificial vagina (AV) from December 2010 until February 2011. Immediately following collection semen was evaluated for initial assessment such as volume and visual motility at $38^{\circ} \mathrm{C}$. The sperm analysis was carried out in CASA laboratory, University Malaya, Malaysia. 


\section{Semen processing}

Semen was processed immediately after collection and was diluted with kampong egg yolk extender or commercial yolk extender. The diluted semen samples were frozen using goat cryopreservation protocols established in University of Malaya, Malaysia. The frozen semen was stored in liquid nitrogen at $-196^{\circ} \mathrm{C}$ until further use.

\section{Sperm parameters}

Fresh and frozen-thawed sperm movement characteristics were analyzed for progressive motility (PM), rapid motility (RM), medium motility (MM), slow motility (SM), static, average path velocity (VAP), progressive velocity (VSL), curvilinear velocity (VCL), amplitude of lateral head displacement (ALH), beat cross frequency (BCF), straightness (STR) and linearity (LIN) using computer assisted semen analysis (CASA, Hamilton-Thorn, USA).

\section{RESULTS AND DISCUSSION}

Three different types of samples such as fresh diluted semen, frozen-thawed "kampong" egg yolk extender (FT-KEY) and frozen-thaw commercial egg yolk extender (FT-CEY) were analyzed using CASA. Sperm movement characteristics such as total motility (TM), progressive motility (PM), rapid motility (RM), medium motility (MM), slow motility (SM), static, average path velocity (VAP), progressive velocity (VSL), curvilinear velocity (VCL), amplitude of lateral head displacement (ALH), beat cross frequency (BCF), straightness (STR) and linearity (LIN) were expressed in mean \pm standard error of mean (SEM) with significant differences $(P \leq 0.05)$.

Fresh diluted semen samples showed better sperm movement characteristics compared to FT-KEY and FT-CEY with the results of highest sperm TM, RM, RM, VAP, VSL, VCL, ALH, STR and LIN; and lowest sperm MM, SM and static (Figures 1; 2).

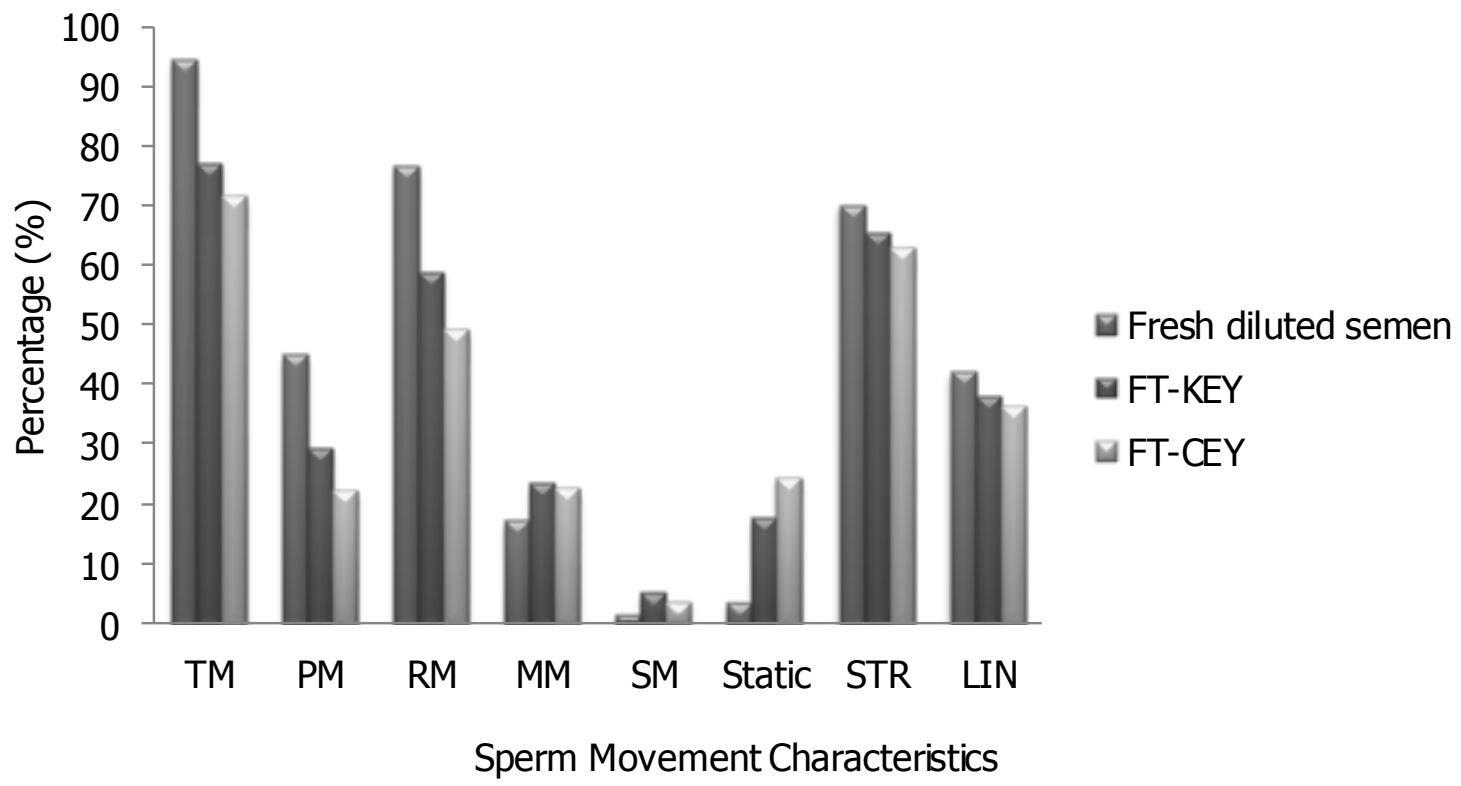

Figure 1. Goat sperm movement characteristics (TM, PM, RM, MM, SM, Static, STR and LIN) of the fresh diluted semen, frozen-thawed "kampong" egg yolk extender (FT-KEY) and frozen-thawed commercial egg yolk extender (FT-CEY). 


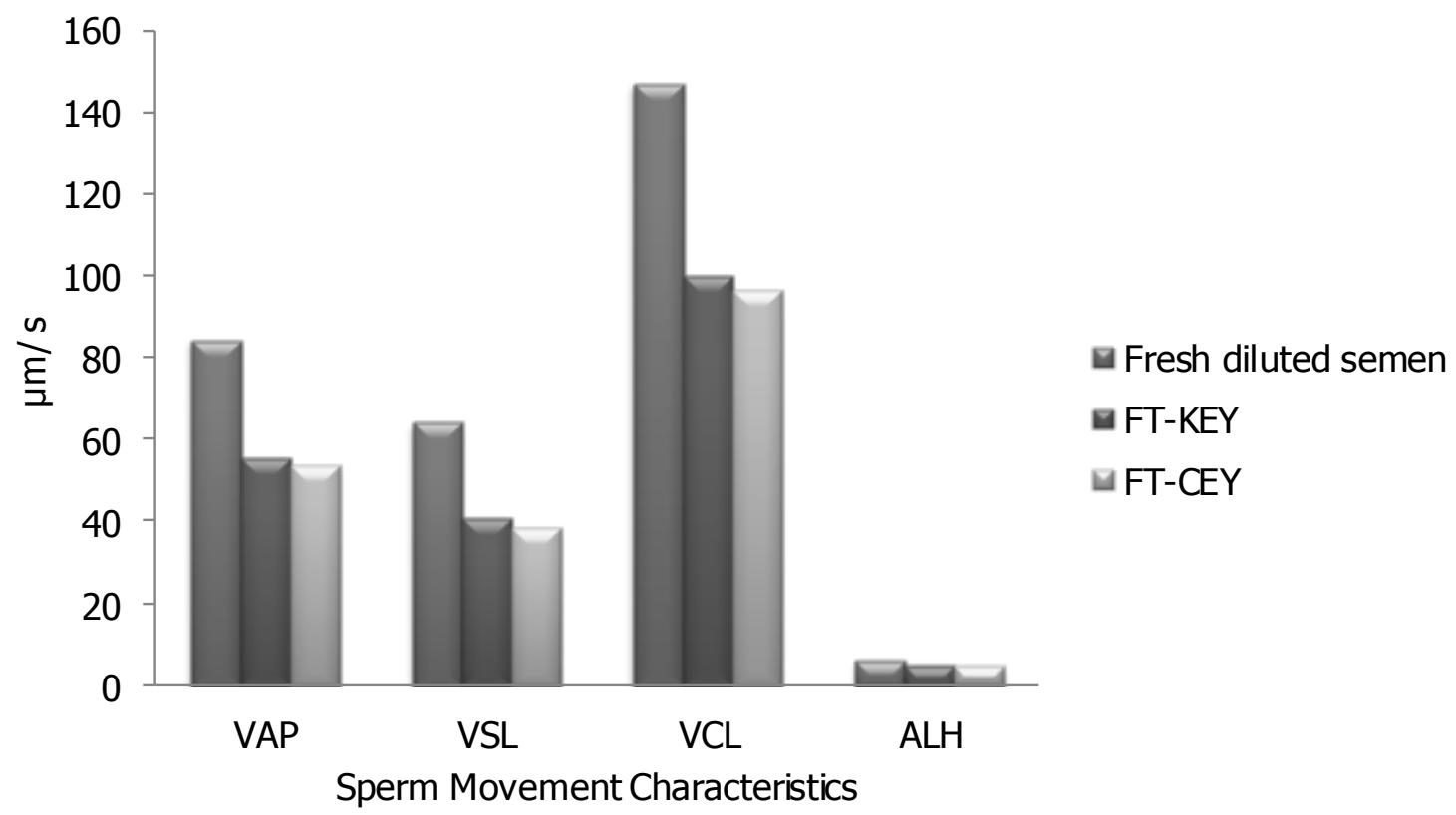

Figure 2. Goat sperm movement characteristics (VAP, VSL, VCL, ALH) of the fresh diluted semen, frozen-thawed "kampong" egg yolk extender (FT-KEY) and frozen-thawed commercial egg yolk extender (FT-CEY).

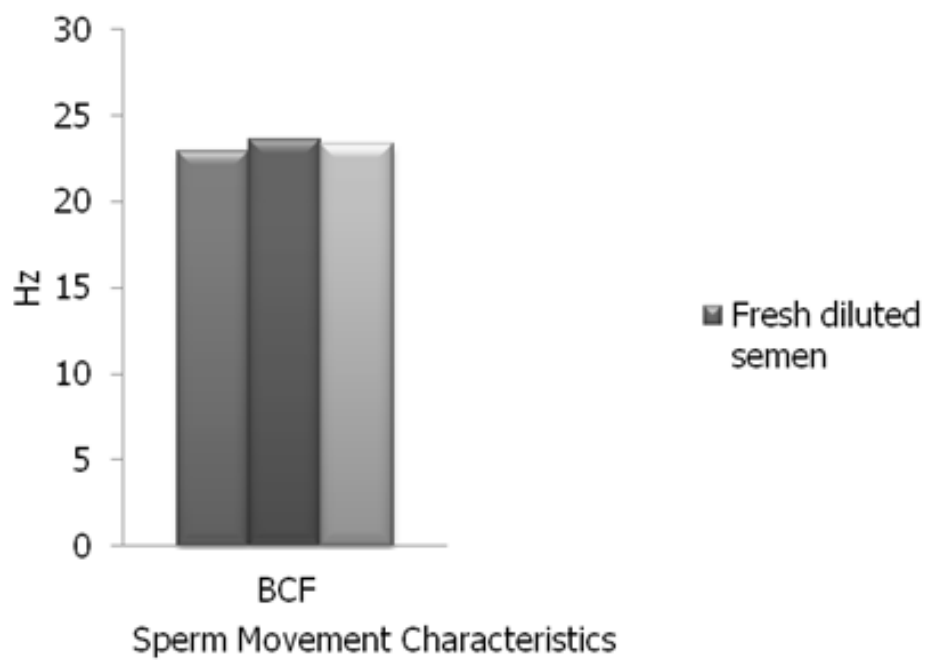

Figure 3. Goat sperm movement characteristics (BCF) of the fresh diluted semen, frozen-thawed "kampong" egg yolk extender (FT-KEY) and frozen-thawed commercial egg yolk extender (FT-CEY)

However, there were no significant differences in the sperm BCF for fresh diluted semen samples compared to FT-KEY and FT-CEY (Figure 3).

There were no significant differences between FT-KEY and FT-CEY in terms of all the sperm movement characteristics. However, FT-KEY showed slightly better sperm movement characteristics compared to FT-CEY with the results higher sperm TM, PM, RM, STR and LIN; lower sperm static (Figure 1; 2). FT-KEY also showed slightly higher in sperm MM and 
SM compared to FT-CEY (Figure 1). The nonsignificant difference between FT-KEY and FTCEY could be due to high standard error of means (SEM) values. The high SEM mainly occurred due to the low number of samples which may confound the result obtained. The number of samples for FT-CEY was lowest ( $\mathrm{n}=$ 7) as compared to FT- KEY $(n=15)$ and fresh diluted semen $(n=14)$. Therefore, FT-CEY showed the highest SEM in all the sperm movement characteristics.

Diluted fresh semen showed significantly higher sperm motility than frozen-thawed sperm in both "kampong" (FT-KEY) and commercial egg yolk (FT-CEY) extenders $\quad(94.79 \pm 1.06 \%$ vs. $77.27 \pm 2.93 \%$ and $71.71 \pm 5.61 \%$, respectively); however, no significant difference was observed on sperm motility between FT-KEY and FTCEY. Similar trends were also observed for other sperm movement characteristics. Deterioration of the sperm movement characteristics in the frozen-thawed samples resulted probably due to dilutions and cryopreservation processes [13]. I

\section{CONCLUSION}

In conclusion, although insignificant differences in movement characteristics of frozen-thawed sperm were observed between both the extenders, apparently kampong chicken egg extender gave better cryoprotection effects compared to the commercial egg extender during goat sperm cryopreservation. However, further refined research is needed to elucidate and improve "kampong" chicken egg extender so that it can be used an alternative choice to other extenders in goat sperm freezing protocol for the industry in the future.

\section{ACKNOWLEDGEMENT}

\section{1}

The authors would like to thank ABEL, Institute of Biological Sciences, Faculty of Science, University of Malaya for providing goats, chemicals, equipment and guidance.

\section{REFERENCES}

1. Holt, W.V. (2000). Fundamental aspects of sperm cryobiology, the importance of species and individual differences. Theriogenology. 53: 47-58.
2. Watson, P.F. (2000). The causes of reduced fertility with cryopreserved semen. Animal Reproduction Science. 60-61, 481-492.

3. Prathalingam, N.S., Holt, W.V., Revell, S.G., Mireuzuk, S., Fleck, R.A. and Watson, P.F. (2006). Impact of antifreeze proteins and antifreeze glycoproteins on bovine sperm during freeze-thaw. Theriogenology. 66: 18941900.

4. Mazur, P. (1984). Freezing of living cells mechanisms and implications. American Journal of Physiology. 247:C125-C142.

5. Alvarez, J.G. and Storey, B.T. (1992). Evidence for increased lipid peroxidative damage and loss of superoxide disumatase activity as a model of sublethal cryodamage to human sperm during cryopreservation. Journal of Andrology. 13: 232-241.

6. Amirat, L., Tainturier, D., Jennaeau, L., Thorin, C., Gerard, O., Courtens, J. L. and Anton, M. (2004). Bull semen in vitro fertility after cyopresrcvation using egg yolk LDL: a comparison with optidyl, a commercial egg yolk extender. Theriogenology. 61: 895-907.

7. Kulaksuz, R., Cebi, C., Akcay, E. and Daskin, A. (2010). The protective effects of egg yolk from different avian species using the cryopreservation of Karayaka ram semen. Small Ruminant Research.88:12-15.

8. Moussa, M., Mannet, V., Trimeche, A., Tamturner, D. and Anton, M. (2002). Low density lipoproteins extracted from hen egg yolk by an easy method: cryoprotective effect on frozen-thawed bull semen. Theriogenology. 57: 16951706.

9. Bergeron, A. and Manjunath, P. (2006). New insights towards understanding the mechanisms of sperm protection by egg yolk and milk. Molecular Reproduction and Development. 73: 1338-1344. 
10. Wall, R.J. and Foote, R.H. (1999). Fertility of bull sperm frozen and stored in clarified egg yolk-Tris-glycerol extender. Journal of Dairy Science. 82:817-821.

11. Clulow, J., Maxwell, W.M.C., Evans, G. and Morris, L.H.A. (2004). A comparison between duck and chicken egg yolk for the cryopreservation of stallion spermatozoa. In the Proceedings of $15^{\text {th }}$ International Congress on Animal Reproduction. Porto Seguro, Brazil. pp. 506.

12. Bathgate, R., Maxwell, W.M.C., Evans, G. (2006). Studies on the effect of supplementing boar semen cryopreservation media with different avian egg yolk types on in vitro postthaw sperm quality. Reproduction of Domestic Animal. 41:68-73.

13. Azawi, O. I., Al-Dahash, S. Y. A. and Juma, F. T. (1993). Effect of different diluents on Shami goat semen. Small Ruminant Research. 9:347-352. 